\title{
COMPARAÇÕES DE MÉTOdOS DE SELEÇÃO EM POPULAÇÕES SIMULADAS DE FRANGOS CAIPIRAS
}

Bruno Eduardo Damacena Silva², Jeferson Corrêa Ribeiro', Wallacy Barbacena Rosa dos Santos ${ }^{3}$, Andreia Santos Cezário ${ }^{3}$, Eliandra Maria Bianchini Oliveira ${ }^{3}$, Tiago Neves Pereira Valente ${ }^{4}$, Aline de Sousa Camargos ${ }^{3}$, Crislaine Messias de Souza ${ }^{3}$

${ }^{1}$ Professor, Departamento de Zootecnia - Instituto Federal Goiano - Campus Morrinhos, GO. ${ }^{2}$ Graduado em Agronomia - Instituto Federal Goiano - campus Morrinhos, GO. ${ }^{3}$ Professor, Departamento de Zootecnia - Instituto Federal Goiano - Campus Morrinhos, GO. ${ }^{4}$ Professor, Instituto Federal Goiano - Campus Posse, GO. E-mail: jeferson.ribeiro@ifgoiano.edu.br

\section{RESUMO}

Nesse trabalho, objetivou-se comparar dois tipos de seleção, BLUP e seleção individual (SI), em frangos caipiras, para a característica peso ao abate, considerando o ganho genético e o valor fenotípico médio como resposta. Foi simulado uma população de frangos caipiras, através do programa Genesys, cuja a população base continha 500 machos e 500 fêmeas, de onde foram escolhidos aleatoriamente 120 machos e 240 fêmeas. A partir da população de seleção gerada, a mesma foi simulada considerando as proporções sexuais de 3, 4, 5 e 6 fêmeas por macho, em dois tipos de seleção: Seleção individual (SI) e BLUP. Em cada método de seleção, utilizou-se o sistema de acasalamento ao acaso entre reprodutores. A seleção foi praticada durante 15 gerações consecutivas e não sobrepostas, com 10 repetições de simulação por geração, para reduzir os efeitos da oscilação genética. Foram avaliadas o ganho genético médio e o valor fenotípico médio em todos os métodos e sistemas de acasalamentos. Os resultados demonstraram que a proporção 6:1 foi superior às outras proporções sexuais nos dois tipos de seleção. Ao compararmos os dois sistemas de seleção, SI foi superior ao BLUP para maior valor fenotípico do peso ao abate. Desta forma, recomenda-se a seleção individual, com proporção sexual de seis fêmeas para cada macho, poderá permitir maiores valores fenotípicos, com menor necessidade de machos.

Palavras-chave: BLUP; ganho genético; peso de abate; seleção individual; valor fenotípico

\section{COMPARISON OF SELECTION METHODS IN SIMULATED POPULATION OF RUSTIC CHICKEN}

\section{ABSTRACT}

The purpose of this study was to compare the two types of selection methods known as BLUP and individual selection (IS) in populations of rustic chicken, evaluating the trait of slaughter weight and considering the averages of genetic gain and phenotypic value as response variables. A population of rustic chicken was simulated in the program Genesys, considering the basal population with 500 males and 500 females, from which was randomly chosen 120 males and 240 females. Then, the selected population was simulated considering the sexual ratios of 3, 4, 5 e 6 females per male in two selection types: Individual Selection (IS) and BLUP. In each selection method, the random mating system between breed chickens was used. Selection was performed during 15 consecutive and non-overlapping generations, with 10 simulation repetitions per generation, in order to decrease the genetic oscillation effects. The average genetic gain and the average phenotypic value in all methods and mating systems were evaluated. The results show that the ratio 6:1 was superior to the other sexual ratios in the two types of selection. When comparing the two selection systems, IS was superior to BLUP for the high phenotypic value of slaughter weight. Thus, the sexual ratio of six females for each male is recommended for individual 
selection, with which will allow high phenotypic values, with lower need of males.

Keywords: BLUP; genetic gain; individual selection; phenotypic value; slaughter weight

\section{INTRODUÇÃO}

O melhoramento genético é praticado corriqueiramente por meio de seleções e acasalamentos. Existem vários métodos de seleção genética, alguns são complementares, outros concorrentes, e a escolha destes métodos dependem das magnitudes e sentidos dos ganhos genéticos que se deseja alcançar, além da facilidade de aplicação dos mesmos.

A seleção direta é a maneira mais fácil e prática de obter ganhos para uma única característica, sendo a resposta à seleção direta a modificação na média original de uma característica da população, em consequência da seleção praticada na própria característica (Paula, 1997). Portanto, numa seleção, quanto maior a pressão de seleção, maior será esse diferencial e, consequentemente, o progresso genético.

Uma alternativa à seleção direta na característica, principalmente aquelas de baixa herdabilidade é através do uso da metodologia dos modelos mistos por meio do BLUP (Best Linear Unbiased Predictor), no qual os reprodutores são selecionados segundo suas informações individuais e, também, segundo as informações colhidas de seus parentes colaterais, ancestrais ou progênies. Esse tipo de seleção visa priorizar o mérito genético individual, em complemento ao valor de suas respectivas famílias, que resulta na obtenção de um valor genético para cada indivíduo, para uma determinada característica de interesse ou para várias características, resultante de uma combinação ótima do valor genético de cada progênie e de cada um dos indivíduos que a compõe (Pires, 1996). Comparar essas duas formas de seleção em aves seria oneroso, além da demanda de tempo e mão-de-obra para obtenção das respostas.

Henderson (1975) afirma que existem três formas para avaliar a eficiência de um método: por meio de análise algébrica, de simulação de dados e de aplicação a um conjunto de dados. A simulação de dados tem sido utilizada em diversas espécies como bovinos de corte (Cunha et al., 2006), bovinos leiteiros (Veerkamp et al., 2002), aves (Reddy, 1996), suínos (de Roo, 2003) e aquicultura (Bentsen; Olesen, 2002), definindo esquemas de seleção ótimos que levam a maiores ganhos genéticos e menores incrementos de endogamia. A partir de modelos biológicos razoáveis para um sistema em particular, pode-se ainda proceder a simulações que permitam avaliações simultâneas de problemas estudados em vários projetos de pesquisa, segundo Rondón et al. (2002). A bioinformática combina conhecimentos multidisciplinares como a biologia, engenharia genética e ciência da computação para processar dados biológicos, com a vantagem de respostas rápidas, precisas e com baixo custo operacional e financeiro.

O objetivo deste trabalho foi comparar dois diferentes tipos de seleção, BLUP e seleção individual, em diferentes proporções sexuais de frangos caipiras, para a característica peso ao abate observando o valor fenotípico médio e o ganho genético como resposta.

\section{MATERIAL E MÉTODOS}

Foram utilizados nesse estudo, dados gerados através de simulação de um genoma semelhante ao de galinhas (Gallus gallus) através do software Genesys (Euclydes, 1996). O genoma foi simulado levando em consideração a característica peso ao abate cuja média inicial utilizada foi fixada em $1500 \mathrm{~g}$, com desvio padrão de $100 \mathrm{~g}$ e herdabilidade igual a 0,30. 0 genoma foi simulado também com 100 locus quantitativos bialélicos, distribuídos ao acaso com 38 cromossomos autossômicos e um sexual, sendo o macho homogamético. Todas as informações iniciais foram simuladas com dados reais.

A partir do genoma gerado, foi simulada uma população base contendo 500 machos e 500 
fêmeas. Da população base, foram escolhidos aleatoriamente 120 machos e 240 fêmeas para constituir a população inicial. A escolha desse número está no fato de ser divisível por 3, 4, 5 e 6, uma vez que na estrutura da população selecionada, foram utilizadas, dentro de cada sistema, as proporções de 3:1, 4:1, 5:1, 6:1 de fêmeas para cada macho. Cada fêmea gerou em média 10 descendentes, sendo considerado o número de descendentes em desequilíbrio, totalizando em 2400 indivíduos. Em seguida foram constituídas populações de seleção, no qual se utilizou dois métodos de seleção: seleção baseada na informação individual (SI) e seleção baseada no melhor preditor linear não-viesado do valor genético (BLUP). Em cada método de seleção, foram utilizados o sistema de acasalamento ao acaso, ou seja, sem restrição de parentesco dos animais selecionados.

As populações de seleção foram simuladas com média de dez descendentes por acasalamento, sendo cada geração composta por 2400 indivíduos dos quais, metade eram machos e a outra metade, constituída por fêmeas.

A seleção foi praticada durante 15 gerações consecutivas e não sobrepostas, com 10 repetições de simulação por geração, para reduzir os efeitos da oscilação genética. Foram avaliadas o ganho genético médio e o valor fenotípico médio em todos os métodos e sistemas de acasalamentos.

Após o processo de simulação, os dados obtidos foram tabulados com o uso do programa Microsoft Office Excel 2010. Após a tabulação das informações, as mesmas foram utilizadas para obtenção de estatísticas descritivas com o uso do programa $\mathrm{R}^{\circledR}$ Development Core Team (2008).

\section{RESULTADOS E DISCUSSÃO}

Dentre as proporções sexuais testadas, através da SI, que podem ser analisados na figura 1 , todas tiveram desempenho semelhante, com valores acima de $2000 \mathrm{~g}$. Observou-se uma leve superioridade da proporção 3:1 e 6:1 em relação às demais para o valor fenotípico. A proporção sexual 5:1 foi a que resultou em menor valor fenotípico. Uma possível explicação para a superioridade da relação 6:1 está na maior pressão de seleção dos machos, ficando esse sexo, com responsabilidade em carregar o progresso genético.

Figura 1. Valor fenotípico médio em gramas para as proporções sexuais de 3:1, 4:1, 5:1 e 6:1 fêmeas por macho em 15 gerações utilizando: (a) seleção individual e (b) BLUP.
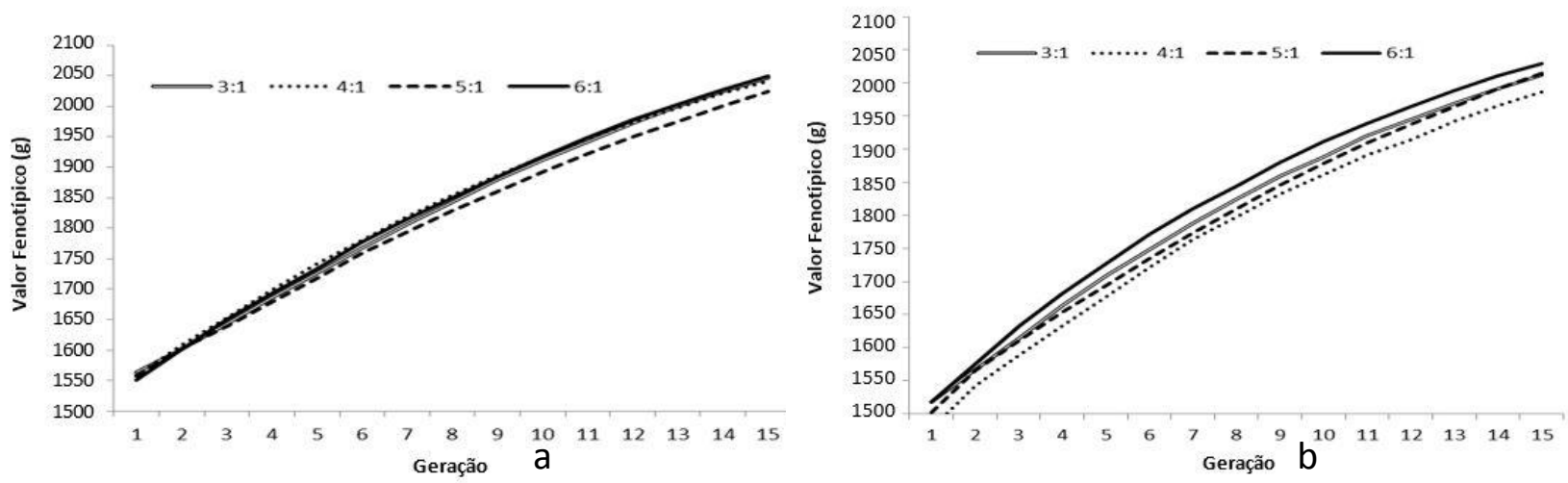

De acordo com Falconer e Mackay (1996), a intensidade de seleção é diretamente relacionada ao ganho genético e inversamente relacionada ao tamanho efetivo de população. 0 tamanho efetivo da população diminui à medida que a proporção de fêmeas para machos é aumentada, ou seja, se o número de fêmeas usadas para cada macho aumenta, o tamanho efetivo diminui. Por consequência, à medida que o número de fêmeas por macho aumenta, aumenta também a taxa de endogamia da população. A proporção 3:1 pode ter resultado em desempenho semelhante ao 6:1 devido à menor taxa de endogamia. A depressão endogâmica ocorre quando a 
taxa de endogamia é alta. Desta forma, o uso de maiores proporções sexuais favorece o aumento da endogamia, o que ocasiona perda da variabilidade genética e também, a diminuição do ganho genético.

No sistema de seleção via BLUP a proporção 6:1 também foi a que obteve melhor resposta, seguidas pelas proporções 3:1 (figura 1). As proporções sexuais de 5:1 e 4:1, nessa ordem, resultaram em menor valor fenotípico, quando comparado às demais proporções. Ao compararmos os dois sistemas de seleção, SI foi superior ao BLUP quanto ao maior valor fenotípico do peso ao abate, com valores observados de até $5 \mathrm{~g}$ acima. Mielenz et al., (1994) mostrou que o grau de vantagem do BLUP sobre SI é determinado pela estrutura do programa de melhoramento e a estratégia do acasalamento, ou seja, nem sempre o uso do BLUP será mais vantajoso. O valor genético do animal através do BLUP é mais acurado, por considerar também a informação de parentesco. Segundo Euclides (1996), o BLUP tem a capacidade de localizar os indivíduos que são portadores de alelos considerados favoráveis e fixar esses alelos, o que torna esse método vantajoso.

O desempenho de cada proporção sexual quanto ao ganho genético, através da seleção individual, pode ser analisado na Figura 2. A proporção sexual com maior ganho genético inicial foi a 6:1. Entretanto essa proporção teve a maior queda e, após 15 gerações, teve desempenho igual às proporções de 3:1 e 5:1. A proporção sexual de 4:1 foi a que resultou em menor ganho genético. Esses valores divergem dos encontrados por Cunha et al. (2006), que obtiveram maiores ganhos genéticos quando o tamanho efetivo era maior. Valores com ganho genético alto no início e queda repentina pode estar ligada ao limite de seleção. Teoricamente quando a resposta à seleção diminui a população pode estar no limite de seleção que, ao ser alcançado, indica que todos os locus foram fixados (Breda et al., 2004). Mesmo assim, o desempenho da proporção 6:1 indica que os ganhos genéticos no início da seleção aceleraram para o aumento do peso dos animais.

Quanto ao BLUP, nesse sistema de seleção, a proporção com maior ganho genético também foi a que utilizou seis fêmeas para cada macho. É sabido que a proporção de 6:1 possui maior intensidade de seleção do que as demais proporções sexuais, ou seja, um menor número de machos é selecionado, e por consequência a pressão de seleção é maior.

A intensidade da seleção é um fator que depende do tamanho efetivo de população para a mesma proporção de selecionados. Conforme observado neste estudo, maiores ganhos genéticos foram obtidos quando houve maior intensidade de seleção nos machos, embora esse ganho não seja sustentado ao longo das gerações devido à maior fixação de alelos não favoráveis ou se o limite de seleção foi atingido.

Figura 2. Ganho genético médio em gramas para as proporções sexuais de 3, 4, 5 e 6 fêmeas por macho em 15 gerações de acasalamento ao acaso: (a) seleção individual e (b) BLUP.

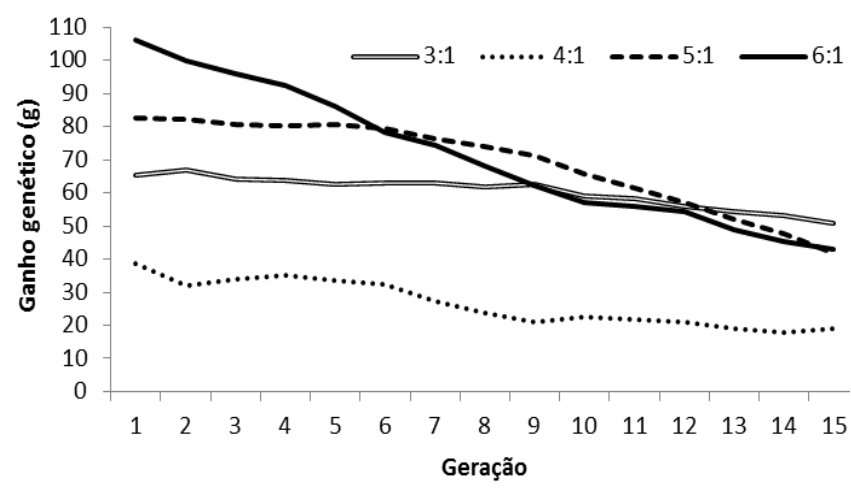

a

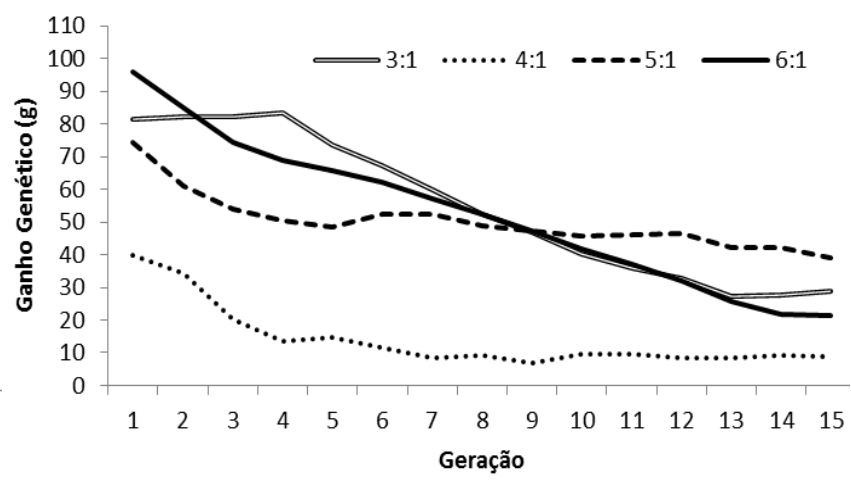

b 
Ao compararmos a seleção individual e via BLUP, podemos perceber uma leve superioridade de até $20 \mathrm{~g}$ no final da $15^{a}$ geração, quando se observa apenas a relação $6: 1$. A grande vantagem do BLUP está na acurácia do valor genético animal, não sendo necessariamente o sistema que implica em maiores ganhos genético. De acordo com Carneiro et al. (2007), o uso da seleção individual resulta em menores taxas de fixação de alelos, tanto favoráveis quanto desfavoráveis. Esses autores afirmam também que o uso da seleção individual provoca menor redução da variabilidade genética, permitindo alcançar maiores ganhos genéticos que o BLUP em características de baixa herdabilidade, após anos de gerações. Entretanto, Cunha et al. (2006) afirma que a curto prazo, a maior acurácia da metodologia BLUP é capaz de otimizar a resposta à seleção, para características de baixa herdabilidade, em função de usar todas as informações disponíveis dos parentes na obtenção da matriz dos numeradores do coeficiente de parentesco de Wright.

\section{CONCLUSÃO}

Recomenda-se utilizar a proporção de seis fêmeas para cada macho, via seleção individual, através acasalamento ao acaso. Essa proporção sexual aliada à seleção individual poderá promover menor custo com o uso de machos, e progresso genético para ganho genético e valor fenotípico médio semelhante às proporções sexuais de 3, 4 e 5 fêmeas por macho, com seleção através do uso do BLUP.

\section{AGRADECIMENTOS}

Somos gratos ao Instituto Federal Goiano campus Morrinhos pelo financiamento dessa pesquisa.

\section{REFERÊNCIAS}

BENTSEN, H.B.; OLESEN, I. Designing aquaculture mass selection programs to avoid high inbreeding rates. Aquaculture. v.204, n. 3-4, p.349-359, 2002. https://doi.org/10.1016/S00448486(01)00846-8

BREDA, F.C.; EUCLYDES, R.F.; PEREIRA, C.S.; TORRES, R.A.; CARNEIRO, P.L.S.; SARMENTO, J.L.R.; TORRES FILHO, R.A.; MOITA, A.K.F. Inbreeding and selection limit in selected population obtained by simulation. Brazilian Journal of Animal Science. v.33, n.6, p.2017-2025, 2004. https://doi.org/10.1590/S1516-35982004000800013

CARNEIRO, P.L.S.; MALHADO, C.H.M.; EUCLYDES, R.F.; CARNEIRO, A.P.S.; CUNHA, E.E. Endogamia, fixação de alelos e limite de seleção em populações selecionadas por métodos tradicionais e associados a marcadores moleculares. Revista Brasileira de Zootecnia, v.36, n.2, p.369-375, 2007. https://doi.org/10.1590/S1516-35982007000200013

CUNHA, E.E.; EUCLYDES, R.F.; TORRES, R.A. et al. Genetic evaluation of beef cattle herds through simulated data. Arquivos Brasileiros de Medicina Veterinária e Zootecnia. v.58, n.3, p.381-387, 2006. https://doi.org/10.1590/S0102-09352006000300015

EUCLYDES, R.F.; MUIR, W.; SILVA, M.A. The Genesys System Simulation. Simpósio Nacional de Melhoramento Animal, 1996, Ribeirão Preto. Anais... Viçosa, 1996. p.303.

FALCONER, D.S.; MACKAY, T.F.C. Introduction to Quantitative Genetics. London/New York: Longmans Green, 1996. 
HENDERSON, C.R. Comparison of alternative sire evaluation methods. Journal of Animal Science, v.41, p. 760-770, 1975. https://doi.org/10.2527/jas1975.413760x

MICROSOFT ${ }^{\circledR}$ OFFICE (2010): Microsoft ${ }^{\circledR}$ Office 2010 Proofing Tools. Microsoft Corporation, Santa Rosa, California.

MIELENZ, N.; GROENEVELD, E.; MULLER, J.; SPILKE, J. Simultaneous estimation of variance and covariances using REML and Henderson 3 in selected population of White Leghorn. British Poultry Science, v.35, p.669-676, 1994. https://doi.org/10.1080/00071669408417733

PAULA, R.C. Avaliação de diferentes critérios de seleção aplicados em melhoramento florestal. 1997. 74f. Tese (Doutorado em Ciência Florestal) - Universidade Federal de Viçosa, Viçosa.

PIRES, I. E. Eficiência da seleção combinada no melhoramento genético de Eucalyptus spp. 1996. 116f. Tese (Doutorado em Melhoramento Genético) - Universidade Federal de Viçosa, Viçosa.

R DEVELOPMENT CORE TEAM (2008). R: A language and environment for statistical computing. $R$ Foundation for Statistical Computing, Vienna, Austria. ISBN 3-900051-07-0, URL http://www.Rproject.org.

REDDY, R.P. Symposium: The effects of long-term selection on growth of poultry. Poultry Science, v.75 p.1164-1167, 1996. https://doi.org/10.3382/ps.0751164

RONDÓN, E.O.O.; MURAKAMI, A.E e SAKAGUTI, E.S. Computer Modeling for Poultry Production and Research. Revista Brasileira de Ciência Avícola, v.4, n.1, p. 199-207, 2002. https://doi.org/10.1590/S1516-635X2002000100001

de ROO, G. A stochastic model to study breeding schemes in a small pig population. Agricultural Systems, v.25, p1-25, 1987. https://doi.org/10.1016/0308-521X(87)90096-5

VEERKAMP, R.F.; DILLON, P.; KELLY, E.; CROMIE, A.R.; GROEN, A.F. Dairy cattle breeding objectives combining yield, survival and calving interval for pasture-based systems in Ireland under different milk quota scenarios. Livestock Production Science, v.76, n.1-2, p.137-151, 2002. https://doi.org/10.1016/S0301-6226(02)00006-4 